\title{
Avaliação da qualidade da manutenção eletromecânica na Companhia de Saneamento Básico do Estado do Piauí
}

\author{
Evaluation of the electromechanical maintenance quality in the Basic Sewage Company of Piauí
}

State

Evaluación de la calidad del mantenimiento electromecánico en la Empresa de Saneamiento Básico del Estado de Piauí

Jorge Luiz de Macêdo

ORCID: https://orcid.org/0000-0003-2857-8026 Universidade Paulista, Brasil E-mail: jorge0316@yahoo.com

Irenilza de Alencar Nääs

ORCID: https://orcid.org/0000-0003-0663-9377 Universidade Paulista, Brasil

E-mail: irenilza.naas@docente.unip.br

Luis Antonio Mendes de Mesquita Araújo

ORCID: https://orcid.org/0000-0001-7638-8652 Universidade Paulista, Brasil

E-mail: irenilza.naas@docente.unip.br

Daiane da Silva Algaves Castelo Branco

ORCID: https://orcid.org/0000-0001-6104-5999

Universidade Paulista, Brasil

E-mail: damalgaves@gmail.com

\begin{abstract}
Resumo
A qualidade do serviço de manutenção eletromecânica aplicada na Companhia de Saneamento Básico do Piauí, na diretoria do interior, ainda é bastante deficitária e necessita de ajustes em seus processos. Neste estudo foi avaliado a qualidade da manutenção eletromecânica aplicada na empresa. Foram realizadas entrevistas, com questionários específicos com os colaboradores e clientes da empresa. Trata-se de uma pesquisa-ação, na qual o pesquisador tem papel ativo no processo de decisão. Utilizou-se a lógica paraconsistente para avaliar a qualidade da manutenção aplicada no processo. Com os resultados encontrados, observou-se que a manutenção corretiva não é a mais adequada e apresenta-se sugestões de mudanças no processo e no estilo de gestão da qualidade para promover a regularidade, melhoria do serviço e desenvolvimento organizacional da empresa.
\end{abstract}

Palavras-chave: Qualidade; Manutenção; Eletromecânica.

\begin{abstract}
The quality of the electromechanical maintenance service applied at the Basic Sanitation Company of Piauí, in the interior board, is still quite deficient and needs to be adjusted in its processes. It is action research in which the researcher has an active role in the decision process. In this study, the quality of electromechanical maintenance applied in the company was evaluated. Interviews were carried out with specific questionnaires with the company's employees and customers. Paraconsistent logic was used to assess the quality of maintenance applied in the process. With the results found, it was observed that corrective maintenance is not the most adequate, and suggestions for changes in the process and the style of quality management are presented to promote regularity, service improvement, and organizational development of the company.
\end{abstract}

Keywords: Quality; Maintenance; Electromechanics.

\section{Resumen}

La calidad del servicio de mantenimiento electromecánico aplicado en la Empresa de Saneamiento Básico de Piauí, en el tablero interior, aún es bastante deficiente y necesita ser ajustada en sus procesos. En este estudio se evaluó la calidad del mantenimiento electromecánico aplicado en la empresa. Se realizaron entrevistas con cuestionarios específicos a los empleados y clientes de la empresa. Es una investigación acción, en la que el investigador tiene un papel activo en el proceso de decisión. Se utilizó lógica paraconsistente para evaluar la calidad del mantenimiento aplicado en el proceso. Con los resultados encontrados, se observó que el mantenimiento correctivo no es el más 
adecuado y se presentan sugerencias de cambios en el proceso y en el estilo de gestión de la calidad para promover la regularidad, la mejora del servicio y el desarrollo organizacional de la empresa.

Palabras clave: Calidad; Mantenimiento; Electromecánica.

\section{Introdução}

O rápido crescimento populacional e a alta taxa de migração para áreas urbanas impõem uma carga pesada na infraestrutura urbana. Particularmente, os sistemas de saneamento básico são alvo de interrupções, causando riscos potenciais à saúde pública. Embora os sistemas de saneamento sejam projetados para lidar com alguns sedimentos e transporte de sólidos, as partículas podem formar depósitos que aumentam o risco de inundação (Fontecha et al., 2020).

Ao longo dos anos, o desenvolvimento da manutenção destes sistemas criou modelos específicos para que fosse possível atuar frente as necessidades das empresas e dos mais variados tipos processos (Denis \& Pontille, 2014). As organizações, em meio a competividade existente, buscam por diferenciação dentro do mercado e querem a garantia de que suas atividades estejam funcionando dentro de sua capacidade, que a entrega de seus produtos e/ou serviços aconteça dentro dos prazos estabelecidos e que a grande maioria, se não todos, estejam dentro do padrão de qualidade estabelecido (Barnes, 2017). O setor de manutenção, por sua vez, é importante e permite que tais objetivos sejam alcançados.

As inovações e tecnologias aplicadas a esse setor, não só de maneira corretiva, mas, principalmente, preventiva, tornou-se um fator decisivo e colabora com a elaboração de modelos estratégicos e tomadas de decisão entre os gestores e colaboradores. Diante dos resultados apresentados por aquelas empresas que fazem esse sistema acontecer de forma eficiente, os serviços de manutenção passaram a ter grande influência e, em muitas situações, são responsáveis pela redução de custos, aumento de lucros, melhorias na operação das máquinas, além de contribuir significativamente com a redução dos impactos ambientais dos projetos e processos (Buser \& Boyer, 2021). Um exemplo muito comum refere-se às empresas de saneamento básico, nas quais existem setores específicos de manutenção eletromecânica, que trabalha com instalações e que envolvem componentes mecânicos e elétricos. Nesta pesquisa, apresenta-se o tipo de manutenção executada, atualmente, na Companhia de Saneamento Básico do Estado do Piauí, especificamente na diretoria do interior, que não atende com satisfação aos clientes, e que durante muito tempo vem apresentando resultados negativos junto à opinião de clientes e colaboradores, além de constante demanda na manutenção de seus equipamentos eletromecânicos, que são danificados frequentemente.

Dessa maneira, com o objetivo de avaliar a qualidade e otimizar a manutenção eletromecânica, foi avaliada a manutenção eletromecânica da empresa, que atende parte significativa do Estado do Piauí.

\section{Referencial Teórico}

\subsection{Tipos de manutenção}

Desde o início da vida humana foi percebida a necessidade de fazer com que as ferramentas utilizadas na caça fossem mantidas em bom estado para que essa atividade ocorresse de forma eficiente. Desde então, existiram diversos episódios históricos que proporcionaram o aprimoramento de ferramentas e a criação de máquinas, estas sendo destinadas a empresas que funcionam para produzir bens e prestar serviços (Netto, 2008). Frente a esse novo cenário outras necessidades foram surgindo e, para que as indústrias funcionassem com maior eficácia o maquinário deveria passar por uma espécie de manutenção para correção de problemas e aperfeiçoamento da atividade (Moreira Neto, 2017).

Macêdo (2013), define manutenção como: "as medidas necessárias para a conservação ou permanência, de alguma coisa ou situação" e ainda como "os cuidados técnicos indispensáveis ao funcionamento regular e permanente de motores e máquinas". Porém, pode-se definir a manutenção como "o conjunto de atividades e recursos aplicados aos sistemas e equipamentos, visando garantir a continuidade de sua função dentro de parâmetros de disponibilidade, de qualidade, de prazo, 
de custos e de vida úteis adequados". A manutenção é caracterizada como um processo, que deve iniciar antes da aquisição e que tem como principal função o prolongamento da vida útil do equipamento ou sistema, para maior conscientização da relação entre manutenção e qualidade do produto, maior pressão para se conseguir alta disponibilidade e confiabilidade da instalação, ao mesmo tempo em que se busca a redução de custos. As alterações estão exigindo novas habilidades do pessoal nas empresas, desde gerentes, passando pelos engenheiros e supervisores, até chegar aos executantes (Cardoso et al., 2014).

O processo de manutenção pode ser classificado de três maneiras: corretivo, preventivo e preditivo. Existem ainda a manutenção produtiva total, a centrada em confiabilidade e a proativa, no entanto apenas as três citadas inicialmente é que serão abordadas nos demais tópicos. A manutenção corretiva é o modelo mais antigo de se realizar manutenção e consiste em atuar somente após a quebra de um equipamento. Por não ser um reparo programado, acaba acarretando prejuízos financeiros, de tempo e produtividade (Trojan et al., 2013). A preventiva também é chamada de manutenção baseada em intervalos/tempo. Ao contrário da manutenção corretiva a manutenção preventiva procura evitar e prevenir antes que a falha efetivamente ocorra (ABNT, 1994). Quanto à manutenção preditiva, esse tipo de manutenção ocorre através da utilização de técnicas que permitem o controle do equipamento através de sistemas operacionais da própria máquina, garantindo assim a qualidade do processo e evitando a realização das manutenções do tipo corretiva e preventiva (Campos \& Rodrigues Filho, 2013).

\subsection{Qualidade da manutenção}

A qualidade nos serviços de manutenção apresenta-se, hoje, como a solução para as organizações se manterem na liderança e captação de mercado, fazendo com que as empresas sejam diferenciadas em seu ramo de atuação. Com a evolução da produção de bens e serviços iniciou-se a preocupação com a qualidade que evoluiu progressivamente na linha do tempo. Essa progressão, por sua vez, chega a ser considerada instrumento de gestão empresarial. A qualidade é a soma de todas as características e propriedades específicas dos bens e serviços oferecidos que atendem as necessidades razoáveis dos clientes, em conjunto com as situações envolvidas na obtenção e uso destes produtos que favoreçam uma existência saudável e autenticamente humana a todos que são afetados (Costa Neto, 2007).

Como garantia de uma boa gestão de qualidade no sistema de manutenção é preciso desenvolver estratégias para que todos os recursos necessários estejam em condições favoráveis de atender aos objetivos de produção e a oferta de serviços. Existem também metodologias e técnicas que podem ser implementadas como forma de buscar a melhoria contínua dos processos e fazer com que os resultados sejam positivos para a organização e para os clientes (Trojan et al., 2013).

Como forma de exemplificar uma dessas metodologias, têm-se a Total Productive Maintenance - TPM ou Manutenção Produtiva Total, que é de origem japonesa e funciona com a intenção de atingir algumas metas, são elas: zero quebra, zero defeito e zero acidente. Quando aplicada de forma correta, proporciona um melhor desempenho no processo produtivo, reduzindo custos e elevando a qualidade dos produtos, além de conceder uma maior segurança aos colaboradores (Melo \& Loos, 2017). Lemos et al. (2011) trazem evidências que reforçam o que foi dito acima, mencionando que "a falta de qualidade da manutenção é um dos geradores de demanda de serviços de manutenção, que consequentemente diminui a disponibilidade, aumenta os custos e diminui a satisfação dos clientes internos e externos. Outro método estratégico bastante difundido em meio ao setor de manutenção é o Planejamento e Controle da Manutenção (PCM), que busca o envolvimento de todos os profissionais da área para definir parâmetros, corrigir falhas e fazer com que os objetivos da empresa sejam alcançados utilizando, basicamente, os recursos disponíveis (Cabral \& Rodrigues, 2017).

A visão da qualidade pode ser vista em oito dimensões específicas: desempenho, complementos, confiabilidade, conformação, assistência técnica, estética e qualidade percebida (Costa Neto, 2007; Correa, 2017). No entanto, mesmo com todas essas alternativas, dentre os segmentos de mercado, alguns ainda possuem certo desfalque em relação ao seu procedimento de manutenção, nas empresas de saneamento básico, por exemplo, a falta de estruturação de um planejamento 
em manutenção contribui para um baixo desempenho das atividades e desequilíbrio empresarial (Lamb et al., 2013). Como forma de amenizar tal efeito, também é importante salientar que os resultados ocorrem em cadeia quando o foco da empresa traz, em primeiro lugar, a qualidade. Para tanto, a organização deve apresentar preocupação geral e constante em todos os níveis e setores. Deve-se iniciar uma clara definição de quais são suas reais possibilidades e o que seria um produto ou serviço de qualidade com base nas necessidades e expectativas dos clientes. Depois disso elaborar o planejamento, associando-o aos aspectos fundamentais como o projeto em si, o desenvolvimento de novos produtos ou serviços e a garantia da qualidade da produção (Costa Neto, 2007).

\subsection{Riscos da manutenção}

Para Calais (2013), risco é o evento ou uma condição incerta que, se ocorrer, tem um efeito em pelo menos um objetivo do trabalho de manutenção. O risco sempre é futuro e pode ter uma ou mais causas, um ou mais impactos e tem origem na incerteza. Deve-se identificá-los e tentar diminuí-los o máximo possível ao longo da execução da atividade. Importante destacar que eles estão relacionados com as demais áreas do conhecimento e devem ser tratados de forma integrada, considerando as melhores práticas de cada área. Os riscos são classificados como: conhecidos, identificados, analisados e considerados na manutenção, ou em qualquer projeto realizado; desconhecidos, que devem ser tratados agilmente, por meio da identificação das causas e adoção de ações preventivas para que o problema não ocorra novamente.

As situações de risco devem ser gerenciadas, tanto com relação a fabricação do produto, quanto as condições de trabalho dos colaboradores. Nessa primeira sua importância se deve ao fato de que é através do seu sucesso que a empresa se mantém dentro do mercado competitivo e a segunda visa o bem-estar e melhor desempenho dos profissionais (Pereira \& Lima, 2012).

A ausência ou má execução do processo de manutenção podem acarretar riscos das mais diversas naturezas. "As revisões periódicas e de manutenção preditiva de máquinas e equipamentos (móveis e fixos) são medidas de controle que garantem um bom funcionamento, aumentam a confiabilidade e minimizam estes riscos” (Martins \& Gomes, 2017).

\section{Metodologia}

Esta é uma pesquisa-ação (Pereira et al., 2018) na qual o pesquisador tem papel ativo no processo de decisão.

\subsection{Caracterização da empresa}

Os órgãos de deliberação superior da empresa definido em seu estatuto social são: Assembleia Geral dos Acionistas, Conselho de Administração e Diretoria Executiva. As contas da empresa são fiscalizadas por um conselho fiscal, pelo Tribunal de Contas do Estado do Piauí e pela Auditoria Geral do Estado. A empresa faz o abastecimento de água e tratamento de esgoto em 157 municípios e 22 povoados do Piauí e tem um patrimônio, apurado por auditoria no último balanço de 2016. São 650 mil ligações de água no Piauí abastecendo uma população de dois milhões das 3,2 milhões de todo o estado. Em Teresina, a empresa tem 247 mil ligações de água que abastece 726 mil habitantes. No Estado do Piauí existem 45 mil ligações de esgotos e em Teresina, 35 mil pontos.

As metas da empresa incluem o abastecimento de água até 2018, cobertura de 80\% do tratamento de esgotos até 2026 e a universalização do fornecimento até 2031. A empresa tem como meta ainda a redução de perdas de água, que hoje está em mais de 50\%, para 25\% até 2026 . 


\subsection{Caracterização do Problema}

Este estudo analisa o tipo de manutenção aplicado atualmente na empresa, especificamente na diretoria do interior. A companhia possui setores específicos no interior, para realização de serviços de manutenção corretiva, ou seja, somente quando o equipamento para por algum motivo é que a equipe de manutenção programa uma intervenção para consertá-lo. Atualmente, o setor de operação e manutenção da empresa está dividido em cinco unidades de negócio e a sede central fica em Teresina. A Superintendência de Desenvolvimento Operacional do Interior conta com 30 colaboradores: um engenheiro eletricista, cinco técnicos em eletromecânica, três bobinadores de motores elétricos, seis mecânicos de manutenção, seis auxiliares técnicos, cinco auxiliares administrativos, dois soldadores e dois torneiros mecânicos. Os serviços executados pela área são os seguintes:

- Retirada e instalação de equipamentos de poços tubulares e nas estações de tratamento de água;

- Realização de ensaios eletromecânicos em todos os equipamentos;

- Limpeza, reaperto e ensaios elétricos em equipamentos das subestações elétricas que fazem parte dos sistemas;

- Reparo, manutenção em painéis de comando, chaves soft-starter, inversores de frequência e instalações elétricas dos sistemas;

- Confecção de peças, mancais de bombeadores, motores elétricos e tubulação hidráulica dos poços e estações de tratamento de água;

- Manutenção em bombas submersas e centrífugas;

- Serviços de solda em geral em tubos e estações de tratamento;

- Manutenção em registros e válvulas;

- Manutenção e rebobinamento de motores elétricos;

- Montagem de sistemas completos de abastecimento de água.

A Figura 1 mostra as quantidades e os tipos de equipamentos recuperados durante os anos de 2012 a 2015 . Pode-se verificar na Figura 1 que houve um acréscimo de motores recuperados, enquanto aparece um decréscimo de bombas recuperadas, e há uma oscilação nos painéis recuperados.

Figura 1 - Recuperação de equipamentos.

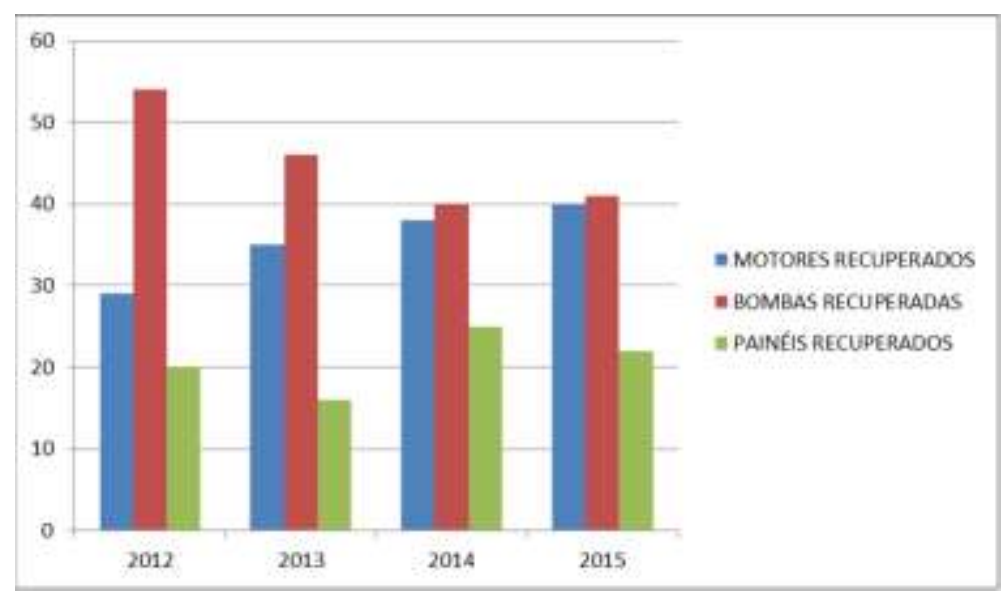

Fonte: Companhia de saneamento básico (2016).

A Figura 2 indica a quantidade e tipos de serviços executados durante os anos de 2012 até 2015. Nota-se que nos serviços executados no período, as atividades de soldagem cresceram até 2014, sendo reduzidas em 2015. Já os serviços de torno e rebobinamento de motor decresceram em 2013 e 2014, entretanto cresceram em 2015. Enquanto a montagem de sistema oscilou entre 2012 e 2015. 
Figura 2 - Tipos de serviços executados.

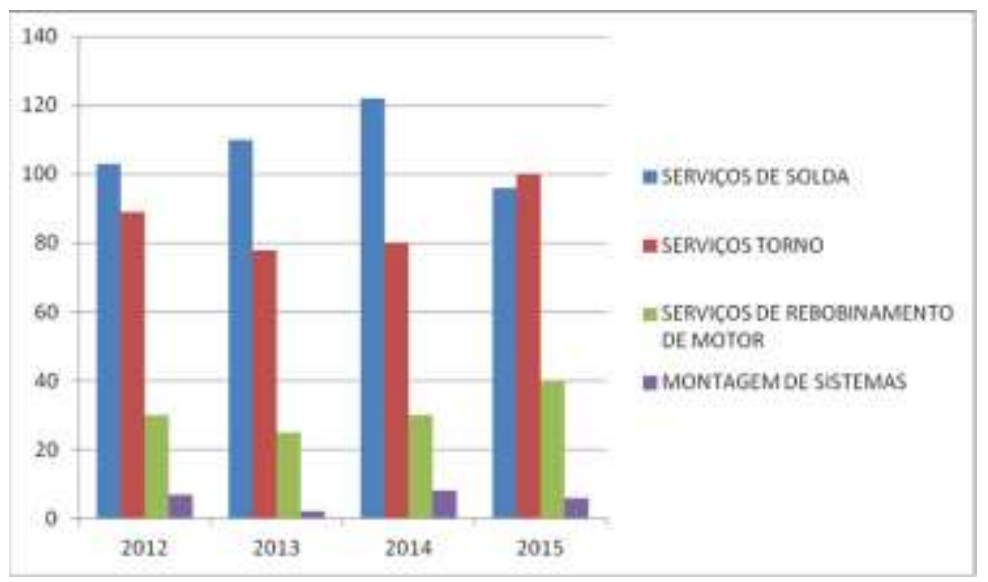

Fonte: Companhia de saneamento básico do Piauí (2016).

\subsection{Procedimentos metodológicos}

Os métodos aplicados para o desenvolvimento da pesquisa foram:

a) Levantamento de informações na empresa, em planilhas da manutenção, serviços executados;

b) Entrevistas com colaboradores e clientes perguntando sobre qualidade do serviço, tempo de execução dos trabalhos, tipo aplicado de manutenção, qual o tipo a ser aplicado;

c) Utilizou-se o Método paraconsistente, com o Software Paradecision para a avaliação das respostas.

Foi utilizado o Software Paradecision para analisar as respostas dos colaboradores da empresa (Figura 3 a, b, c). A lógica paraconsistente é apropriada para operar sistemas inconsistentes, eliminando as contradições (Carvalho \& Abe, 2011). As decisões, na maioria das vezes, são baseadas em aspectos subjetivos, que não tem valor ou medida exata, preconiza a opinião do meio ao se relacionar. A Lógica Paraconsistente revoga o princípio da não contradição, e admite o tratamento de sinais contraditórios na sua estrutura interna.

Na Figura 3a é mostrada a realização de uma avaliação da qualidade do serviço de manutenção desenvolvida na empresa. As Figura 3b e 3c, por sua vez, mostram o processamento da avaliação do tipo de manutenção aplicada na empresa e apresentadas listadas nos resultados.

Figura 3 - Software aplicado nas condições da empresa.

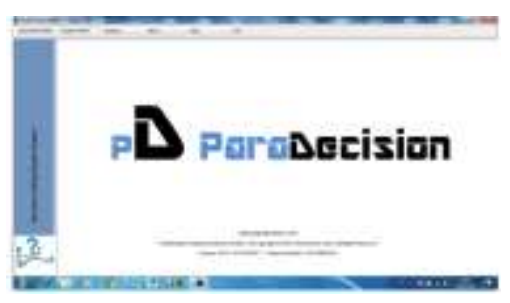

a

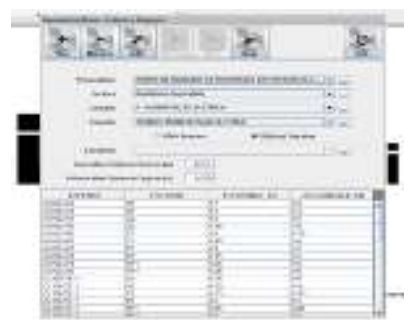

b

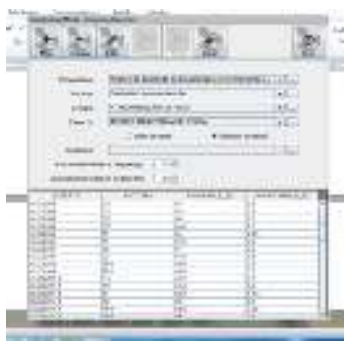

$\mathrm{c}$

Fonte: Autores.

Na Figura 4 têm-se a avaliação do tempo de execução da manutenção aplicada na empresa. E por fim, a Figura 5, que mostra a obtenção da compilação dos resultados aplicados especificamente em cada um dos parâmetros. A partir dessas informações é possível fazer uma avaliação mais aprofundada da situação do desempenho da atividade de manutenção na 
empresa.

Figura 4 - Avaliação do tempo de execução da manutenção

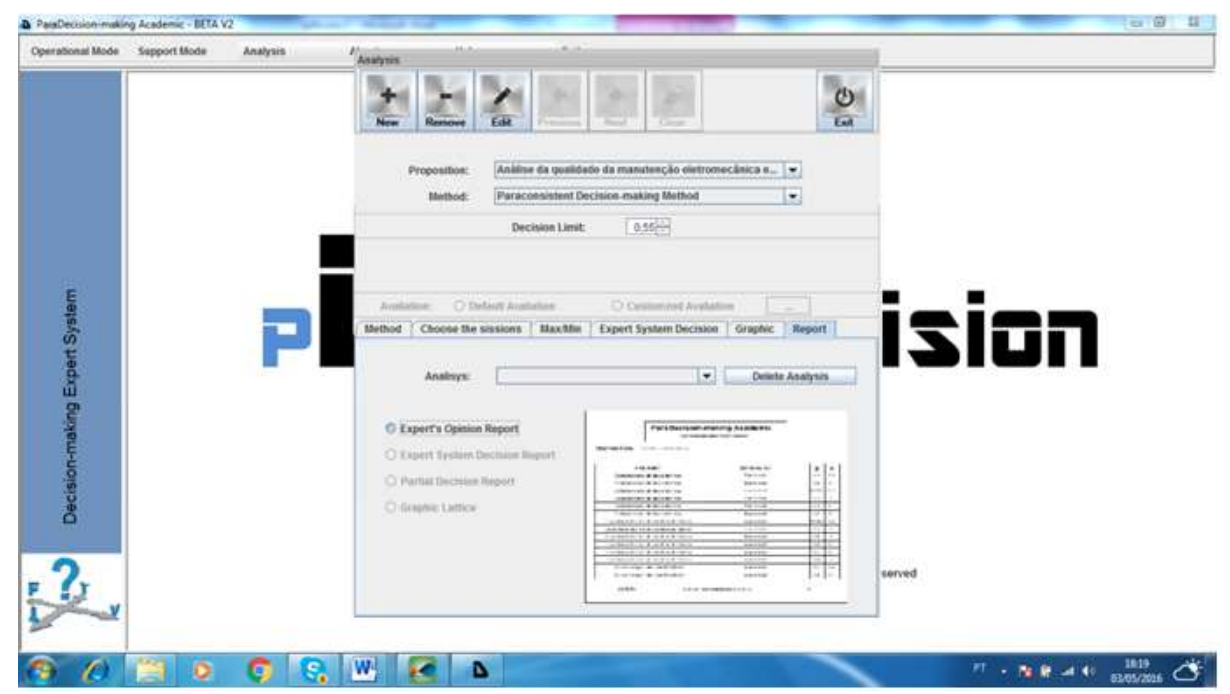

Fonte: Paradecision: (2016).

Figura 5 - Compilação dos dados obtidos

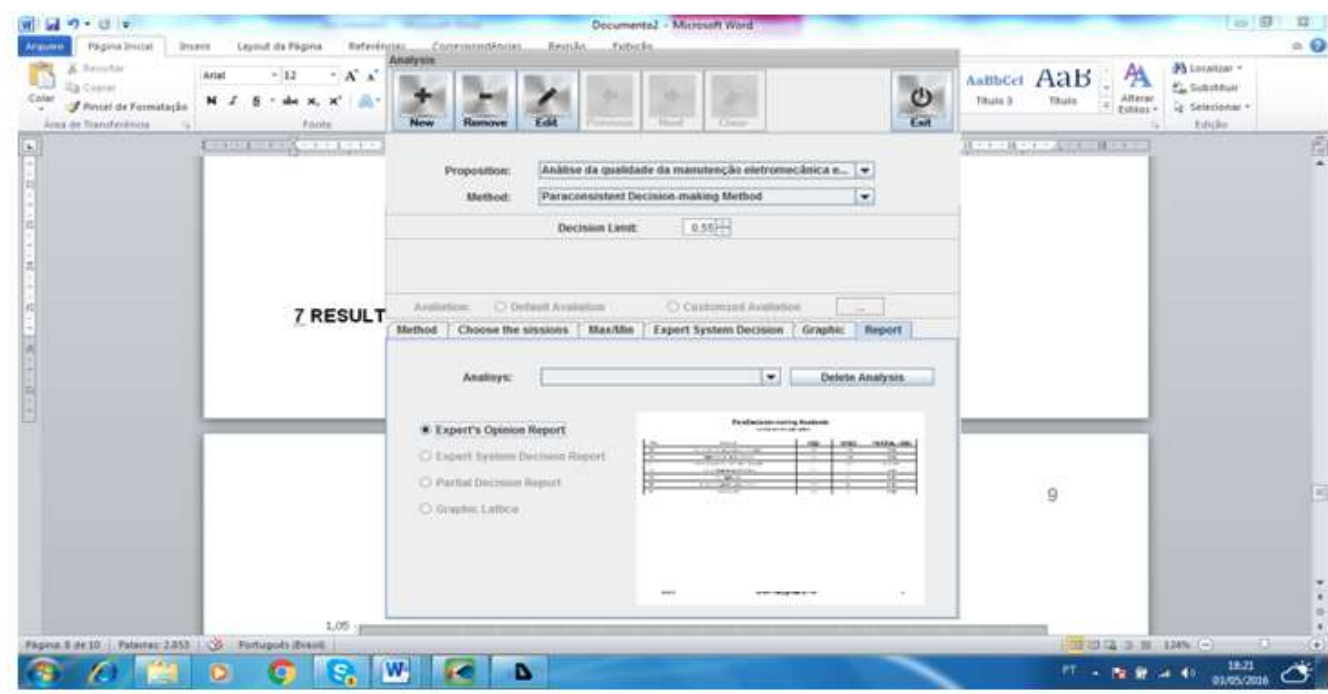

Fonte: Paradecision: (2016).

\section{Resultados e Discussão}

A partir da compilação dos dados foi possível gerar um gráfico (Figura 5 a), que apresenta um resultado não satisfatório para os parâmetros avaliados, uma vez que estes se situaram fora do lattice da paradecisão. O algoritmo Paraanalisador, além dos quatro estados extremos, é possível determinar mais oito estados não extremos. Observa-se, então, que com o algoritmo Para-analisador (Figura 5b), além dos quatro estados extremos, é possível determinar mais oito estados não extremos. Na Tabela 1 indicam-se as regiões que representam os estados não extremos neste algoritmo. 
Figura 5 - Resultados da análise de paradecisão aplicado.

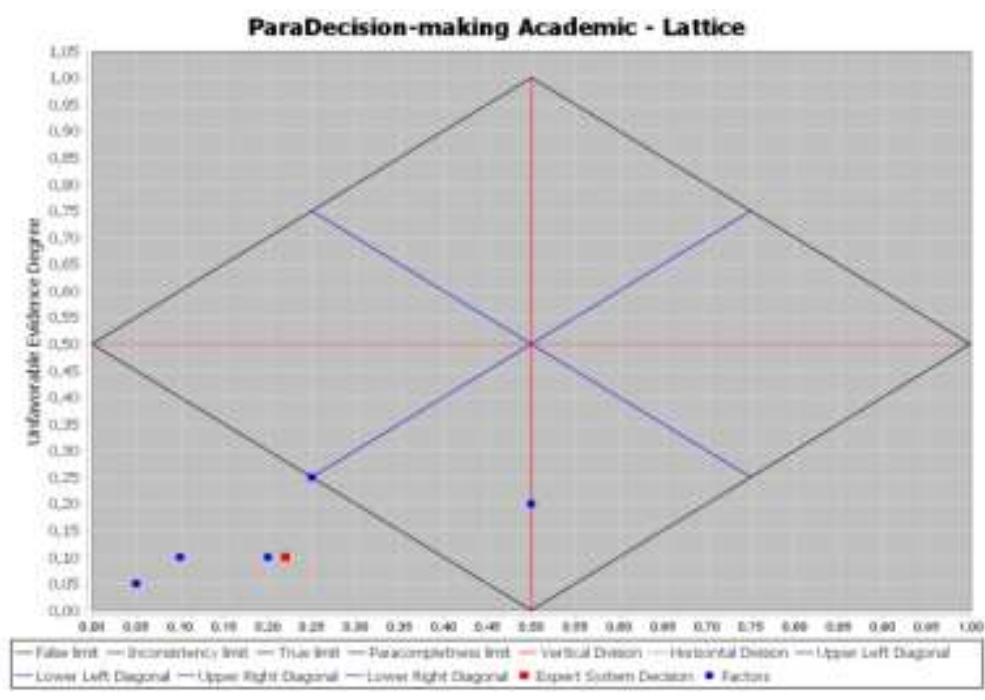

a

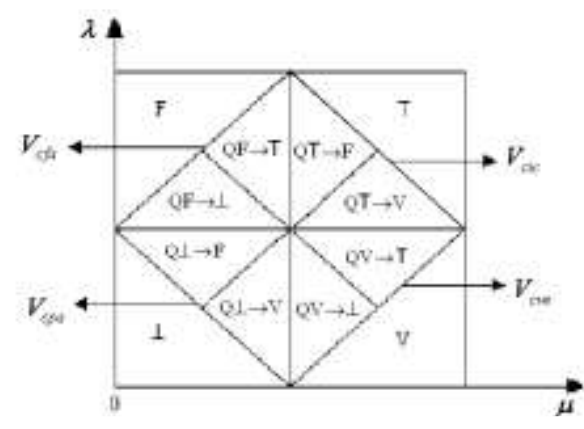

b

Fonte: Autores.

Tabela 1 - Representação de Estados Não Extremos da Lógica Paraconsistente.

\begin{tabular}{ll}
\hline Símbolo & Descrição \\
\hline $\mathbf{Q V} \rightarrow \mathbf{T}$ & Quase verdadeiro tendendo para o inconsistente \\
\hline $\mathbf{Q V} \rightarrow \perp$ & Quase verdadeiro tendendo para Paracompleteza \\
\hline $\mathbf{Q F} \rightarrow \mathbf{T}$ & Quase falso tendendo para o inconsistente \\
\hline $\mathbf{Q F} \rightarrow \perp$ & Quase falso tendendo para Paracompleteza \\
\hline $\mathbf{Q T} \rightarrow \mathbf{V}$ & Quase inconsistente tendendo a verdadeiro \\
\hline $\mathbf{Q T} \rightarrow \mathbf{F}$ & Quase inconsistente tendendo a falso \\
\hline $\mathbf{Q} \perp \rightarrow \mathbf{V}$ & Quase Paracompleteza tendendo para verdadeiro \\
\hline $\mathbf{Q} \perp \rightarrow \mathbf{F}$ & Quase Paracompleteza tendendo para falso \\
\hline
\end{tabular}

Fonte: Abe et al. (2015)

Aplicados os questionários usando a lógica paraconsistente os resultados encontrados foram significativos para a tomada de decisão na empresa e observou-se os seguintes problemas:

a) Somente quando o equipamento é danificado e para de funcionar é que a equipe de manutenção é avisada pelo escritório local e vai até o local para realizar a retirada do equipamento, sendo o mesmo transportado para Teresina para a devida manutenção. Às vezes, o sistema afetado fica sem equipamento e deixa de abastecer em períodos prolongados, o que traz prejuízo financeiro à empresa;

b) Equipamentos reserva servem para substituir outros sistemas também, por isso sistemas ficam sem equipamentos extras;

c) O tempo que é levado para recuperar o equipamento danificado é bastante prolongado devido ao extenso percurso até a oficina eletromecânica, em Teresina;

d) Os gastos com diárias e muitas vezes com o retrabalho eleva bastante os custos de manutenção e operação;

e) O treinamento dos colaboradores não acontece constantemente, prejudicando a qualidade do trabalho realizado 
nos sistemas;

f) Muitas vezes sistemas inteiros deixam de funcionar normalmente, prejudicando o abastecimento de água e deixando de arrecadar para a empresa;

g) O cliente apresenta-se insatisfeito quanto ao tempo gasto para o atendimento;

h) Falta a implantação de um sistema de gestão da qualidade efetivo.

A principal alteração que a empresa deve realizar se refere a qualidade de seus serviços de manutenção e a conscientização de seu corpo funcional. Pois como já foi abordado, o fator qualidade dentro das organizações vem ganhando muitos destaques positivos, quando se observam os resultados. Tais resultados confirmam os estudos de Buser \& Boyer (2021) quando investigaram a manutenção da infraestrutura abaixo do solo de serviços em Paris e baseando-se nessa compreensão mais ampla de qualidade da manutenção e reparo de infraestrutura como uma forma de cuidado no sentido de que a infraestrutura precisa de atenção para evitar danos. O foco dado pelos autores ressalta a importância da atividade humana na manutenção de diferentes tipos de infraestrutura.

Por outro lado, em contraste com a noção preventiva de manutenção, o reparo é geralmente entendido como centrado naqueles momentos de falha e interrupção quando um objeto ou infraestrutura quebra e torna-se presente à mão (Lamb et al., 2013). No entanto, Denis e Pontille (2014) estabelecem outras atividades onde o reparo como manutenção reflete aquelas práticas associadas à resolução de problemas e ajustes sem grande revisão sistêmica. Em contraste, o reparo como transformação envolve um reordenamento mais abrangente ou estrutural das relações que podem desafiar as formas aceitas de operação. Para Fontecha et al. (2020), as decisões para manter as infraestruturas e o controle de problemas estruturais foram incorporados às dependências contextuais e relações de poder que restringiram as possibilidades de uma transformação tão necessária da abordagem da cidade para os riscos.

Observou-se que a pesquisa proporcionou à empresa, condições para uma melhor compreensão do comportamento dos custos e das oportunidades para a redução destes na manutenção eletromecânica, podendo ser aplicado em outras unidades da empresa ou em outras organizações, bastando para isso, que alguns ajustes ou adaptações, como a implantação do sistema de gestão da qualidade (SGQ), sejam realizados para a melhoria dos resultados.

\section{Conclusões}

A partir da avaliação da situação do sistema de manutenção da empresa de saneamento básico em Teresina - PI, é possível concluir que o sistema de manutenção corretiva aplicado na empresa não atende às expectativas de clientes e colaboradores, criando gargalos específicos na produção, recuperação de equipamentos e serviços executados na empresa. Frente ao estudo que foi realizado é importante salientar que a empresa necessita realizar certa modificação e aplicar um plano de manutenção específico voltado para a manutenção eletromecânica preventiva, fazendo com que imprevistos sejam evitados e os riscos de parada minimizados. Para estudos futuros recomenda-se ampliar o período estudado, bem como avaliar a implantação de um sistema de qualidade dos processos.

\section{Referências}

Abe, J. M. (2015). Paraconsistent intelligent-based systems: New trends in the applications of paraconsistency (Vol. 94). Springer.

Ballestero-Alvarez, M. E. (2012). Gestão da qualidade, produção e operações. (2a ed.), Atlas.

Barnes, B. (2017). States of Maintenance: Power, politics, and Egypt's irrigation infrastructure. Environment and Planning D, 35, $146-64$.

Buser, M., \& Boyer, K. (2021). Care goes underground: thinking through relations of care in the maintenance and repair of urban water infrastructures. Cultural Geographies 28, 73-90. 
Cabral, D. A., \& Rodrigues, L. M. (2019). A implantação do PCM como estratégia para aumento de produtividade e redução de custos: pesquisa-ação em uma oficina portuária. https://www.aedb.br/seget/arquivos/artigos17/15825152.pdf.

Calais, J. (2013). Importância do correto funcionamento de equipamentos eletromecânicos numa empresa de tratamento e abastecimento de águas. Corvilha: Dissertação de mestrado em Engenharia Electromecânica, Universidade da Beira Interior.

Campos, F. H. L. F. de, \& Rodrigues Filho, O. (2013). Aplicação de manutenção preditiva em um sistema de descarregamento de caminhões e vagões tanque. TCC Curso de Tecnologia em Mecatrônica Industrial, Universidade Tecnológica Federal do Paraná, Curitiba.

Cardoso, G. T., Shimote, L. E. C., \& Puglisi, J. F. S. (2014). Gestão da Manutenção. https://www.passeidireto.com/arquivo/19567220/gestao-da-manutencao.

Carvalho, F. R. D., \& Abe, J. M. (2011). Tomadas de Decisão com Ferramentas da Lógica Paraconsistente Anotada. Editora Edgard Blucher Ltda.

Correa, C. (2017). Vicente Falconi: O que importa é resultado. Primeira Pessoa.

Denis J., \& Pontille, D. (2014). Maintenance work and the performativity of urban inscriptions: The case of Paris subway signs. Environment and Planning D: Society and Space, 32,404-16.

Ferreira, A. B. (2009). Novo Dicionário Aurélio da Língua Portuguesa. (4a ed.), Positivo.

Fontecha, J. E., Guaje, O. O., Duque, D. et al. (2020). Combined maintenance and routing optimization for large-scale sewage cleaning. Annual Operational Research 286, 441-474.

Kardec, A., \& Nascif, J. (2001). Manutenção: Função Estratégica. (3a ed.), Qualitymark.

Lamb, M. A. et al. (2013). Modelo de planejamento e controle da manutenção para empresas de saneamento básico http://abepro.org.br/biblioteca/enegep2013_TN_STO_177_011_22655.pdf.

Lemos, M. A., Albernaz, C. M. R. M., Carvalho, R. A. de. (2011). Qualidade na Manutenção. http://www.abepro.org.br/biblioteca/eneg ep2011_tn_sto_135_859_18052.pdf.

Macêdo, J. L. (2013). A implantação do processo de manutenção preventiva eletromecânica móvel na unidade de negócio do entorno-genen da companhia de saneamento básico do Piauí. Revista Saneas, 49, 1-12.

Mello Neto, F. de C., Peres, M. L., \& Cardoso, I. A. P. (2011). A importância da manutenção para o negócio. http://www.abepro.org.br/bi blioteca/enegep2011_TN_STO_135_859_18427.pdf.

Melo, F. T., \& Loos, M. J. (2017). Análise da metodologia da Manutenção Produtiva Total (TPM): Estudo de caso. Revista Espacios 39, 1-13.

Moreira Neto, T. C. (2017). A história da evolução do sistema de gestão de manutenção. https://www.webartigos.com/artigos/a-historia-da-evolucao-dosistema-de-gestao-de-manutencao/75650.

Neto, P. L. O. C. (2007). Qualidade e Competência nas Decisões. Edgard Blucher, 2007.

Netto, W. A. C. (2008). A importância e a aplicabilidade da manutenção produtiva total (TPM) nas indústrias. Monografia (Especialização), Curso de Engenharia de Produção, Universidade Federal de Juiz de Fora. http://www.ufjf.br/ep/files/2010/05/Wady-UFJF-Engenharia-Monografia.pdf

Pereira, A.S., \& et al. (2018). Metodologia da pesquisa científica. UFSM.

Pereira, M. J. (2009). Engenharia de Manutenção: Teoria e Prática. Moderna.

Trojan, F., Marçal, R. F. M., \& Baran, L. R. (2013). Classificação dos tipos de manutenção pelo método de análise multicritério ELECTRE TRI. http://www.din.uem.br/ ademir/sbpo/sbpo2013/pdf/arq0338.pdf. 